\title{
On Continuity of Functions between Vector Metric Spaces
}

\author{
Cüneyt Çevik \\ Department of Mathematics, Faculty of Science, Gazi University, Teknikokullar, 06500 Ankara, Turkey \\ Correspondence should be addressed to Cüneyt Çevik; ccevik@gazi.edu.tr
}

Received 17 August 2014; Accepted 8 October 2014; Published 12 November 2014

Academic Editor: Han Ju Lee

Copyright (C) 2014 Cüneyt Cevik. This is an open access article distributed under the Creative Commons Attribution License, which permits unrestricted use, distribution, and reproduction in any medium, provided the original work is properly cited.

We introduce vectorial and topological continuities for functions defined on vector metric spaces and illustrate spaces of such functions. Also, we describe some fundamental classes of vector valued functions and extension theorems.

\section{Introduction and Preliminaries}

Let $E$ be a Riesz space. If every nonempty bounded above (countable) subset of $E$ has a supremum, then $E$ is called Dedekind $(\sigma-)$ complete. A subset in $E$ is called order bounded if it is bounded both above and below. We write $a_{n} \downarrow a$ if $\left(a_{n}\right)$ is a decreasing sequence in $E$ such that inf $a_{n}=a$. The Riesz space $E$ is said to be Archimedean if $n^{-1} a \downarrow 0$ holds for every $a \in E_{+}$. A sequence $\left(b_{n}\right)$ is said to be o-convergent (or order convergent) to $b$ if there is a sequence $\left(a_{n}\right)$ in $E$ such that $a_{n} \downarrow 0$ and $\left|b_{n}-b\right| \leq a_{n}$ for all $n$, where $|a|=a \vee(-a)$ for any $a \in E$. We will denote this order convergence by $b_{n} \stackrel{o}{\rightarrow} b$. Furthermore, a sequence $\left(b_{n}\right)$ is said to be $o$-Cauchy if there exists a sequence $\left(a_{n}\right)$ in $E$ such that $a_{n} \downarrow 0$ and $\left|b_{n}-b_{n+p}\right| \leq a_{n}$ for all $n$ and $p$. The Riesz space $E$ is said to be $o$-Cauchy complete if every $o$-Cauchy sequence is $o$-convergent.

An operator $T: E \rightarrow F$ between two Riesz spaces is positive if $T(x) \geq 0$ for all $x \geq 0$. The operator $T$ is said to be order bounded if it maps order bounded subsets of $E$ to order bounded subsets of $F$. The operator $T$ is called $\sigma$ order continuous if $x_{n} \stackrel{o}{\rightarrow} 0$ in $E$ implies $T\left(x_{n}\right) \stackrel{o}{\rightarrow} 0$ in $F$. Every $\sigma$-order continuous operator is order bounded. If $T(x \vee y)=T(x) \vee T(y)$ for all $x, y \in E$, the operator $T$ is a lattice homomorphism. For further information about Riesz spaces and the operators on Riesz spaces, we refer to $[1,2]$.

In [3], a vector metric space is defined with a distance map having values in a Riesz space, and some results in metric space theory are generalized to vector metric space theory. Some fixed point theorems in vector metric spaces are given in [3-7]. Actually, the study of metric spaces having value on a vector space has started by Zabrejko in [8]. The distance map in the sense of Zabrejko takes values from an ordered vector space. We use the structure of lattice with the vector metrics having values in Riesz spaces; then we have new results as mentioned above. This paper is on some concepts and related results about continuity in vector metric spaces.

Definition 1. Let $X$ be a nonempty set and let $E$ be a Riesz space. The function $d: X \times X \rightarrow E$ is said to be a vector metric (or $E$-metric) if it satisfies the following properties:

$(\operatorname{vml}) d(x, y)=0$ if and only if $x=y$,

$(\operatorname{vm} 2) d(x, y) \leq d(x, z)+d(y, z)$ for all $x, y, z \in X$.

Also the triple $(X, d, E)$ is said to be vector metric space.

Definition 2. Let $(X, d, E)$ be a vector metric space.

(a) A sequence $\left(x_{n}\right)$ in $X$ is vectorially convergent (or is $E$-convergent) to some $x \in X$, if there is a sequence $\left(a_{n}\right)$ in $E$ such that $a_{n} \downarrow 0$ and $d\left(x_{n}, x\right) \leq a_{n}$ for all $n$. We will denote this vectorial convergence by $x_{n} \stackrel{d, E}{\longrightarrow}$ $x$.

(b) A sequence $\left(x_{n}\right)$ in $X$ is called $E$-Cauchy sequence whenever there exists a sequence $\left(a_{n}\right)$ in $E$ such that $a_{n} \downarrow 0$ and $d\left(x_{n}, x_{n+p}\right) \leq a_{n}$ for all $n$ and $p$.

(c) The vector metric space $X$ is called $E$-complete if each $E$-Cauchy sequence in $X$ is $E$-convergent to a limit in $X$. 
One of the main goals of this paper is to demonstrate the properties of functions on vector metric spaces in a context more general than the continuity in metric analysis. Hence, the properties of Riesz spaces will be the tools for the study of continuity of vector valued functions. The result we get from here is continuity in general sense, an order property rather than a topological feature.

In Section 2, we consider two types of continuity on vector metric spaces. This approach distinguishes continuities vectorially and topologically. Moreover, vectorial continuity examples are given and the relationship between vectorial continuity of a function and its graph is demonstrated. In Section 3, equivalent vector metrics, vectorial isometry, vectorial homeomorphism definitions, and examples related to these concepts are given. In Section 4, uniform continuity is discussed and some extension theorems for functions defined on vector metric spaces are given. Finally, in Section 5, a uniform limit theorem on a vector metric space is given, and the structure of vectorial continuous function spaces is demonstrated.

\section{Topological and Vectorial Continuity}

We now introduce two types of continuity in vector metric spaces. For two elements $a$ and $b$ in a Riesz space, we write $a<b$ to indicate that $a \leq b$ but $a \neq b$.

Definition 3. Let $(X, d, E)$ and $(Y, \rho, F)$ be vector metric spaces, and let $x \in X$.

(a) A function $f: X \rightarrow Y$ is said to be topologically continuous at $x$ if for every $b>0$ in $F$ there exists some $a$ in $E$ such that $\rho(f(x), f(y))<b$ whenever $y \in X$ and $d(x, y)<a$. The function $f$ is said to be topologically continuous if it is topologically continuous at each point of $X$.

(b) A function $f: X \rightarrow Y$ is said to be vectorially continuous at $x$ if $x_{n} \stackrel{d, E}{\longrightarrow} x$ in $X$ implies $f\left(x_{n}\right) \stackrel{\rho, F}{\longrightarrow}$ $f(x)$ in $Y$. The function $f$ is said to be vectorially continuous if it is vectorially continuous at each point of $X$.

Theorem 4. Let $(X, d, E)$ and $(Y, \rho, F)$ be vector metric spaces where $F$ is Archimedean. If a function $f: X \rightarrow Y$ is topologically continuous, then $f$ is vectorially continuous.

Proof. Suppose that $x_{n} \stackrel{d, E}{\longrightarrow} x$. Then there exists a sequence $\left(a_{n}\right)$ in $E$ such that $a_{n} \downarrow 0$ and $d\left(x_{n}, x\right) \leq a_{n}$ for all $n$. Let $b$ be any nonzero positive element in $F$. By topological continuity of $f$ at $x$, there exist elements $b_{n}=b_{n}((1 / n) b ; x)$ in $E$ such that $d\left(x, x_{n}\right)<b_{n}$ implies $\rho\left(f(x), f\left(x_{n}\right)\right)<(1 / n) b$ for all $n$. Then there exist elements $c_{n}=a_{n} \vee b_{n}$ in $E$ such that $d\left(x_{n}, x\right) \leq$ $a_{n}<c_{n}$ implies $\rho\left(f\left(x_{n}\right), f(x)\right)<(1 / n) b$ for all $n$. Since $F$ is Archimedean, $(1 / n) b \downarrow 0$. Hence, $f\left(x_{n}\right) \stackrel{\rho, F}{\longrightarrow} f(x)$.

Vectorially continuous functions have a number of nice characterizations.
Corollary 5. For a function $f: X \rightarrow Y$ between two vector metric spaces $(X, d, E)$ and $(Y, \rho, F)$ the following statements hold.

(a) If $F$ is Dedekind $\sigma$-complete and $f$ is vectorially continuous, then $\rho\left(f\left(x_{n}\right), f(x)\right) \downarrow 0$ whenever $d\left(x_{n}, x\right) \downarrow 0$.

(b) If $E$ is Dedekind $\sigma$-complete and $\rho\left(f\left(x_{n}\right), f(x)\right) \downarrow$ 0 whenever $d\left(x_{n}, x\right) \downarrow 0$, then the function $f$ is vectorially continuous.

(c) Suppose that $E$ and $F$ are Dedekind $\sigma$-complete. Then, the function $f$ is vectorially continuous if and only if $\rho\left(f\left(x_{n}\right), f(x)\right) \downarrow 0$ whenever $d\left(x_{n}, x\right) \downarrow 0$.

Proof. (a) If $d\left(x_{n}, x\right) \downarrow 0$, then $x_{n} \stackrel{d, E}{\longrightarrow} x$. By the vectorial continuity of the function $f$, there is a sequence $\left(b_{n}\right)$ in $F$ such that $b_{n} \downarrow 0$ and $\rho\left(f\left(x_{n}\right), f(x)\right) \leq b_{n}$ for all $n$. Since $f$ is Dedekind $\sigma$-complete, $\rho\left(f\left(x_{n}\right), f(x)\right) \downarrow 0$ holds.

(b) Let $x_{n} \stackrel{d, E}{\longrightarrow} x$ in $X$. Then there is a sequence $\left(a_{n}\right)$ in $E$ such that $a_{n} \downarrow 0$ and $d\left(x_{n}, x\right) \leq a_{n}$ for all $n$. Since $E$ is Dedekind $\sigma$-complete, $d\left(x_{n}, x\right) \downarrow 0$ holds. By the hypothesis, $\rho\left(f\left(x_{n}\right), f(x)\right) \downarrow 0$, and so $f\left(x_{n}\right) \stackrel{\rho, F}{\longrightarrow} f(x)$ in $Y$.

(c) Proof is a consequence of (a) and (b).

Example 6. Let $(X, d, E)$ be a vector metric space. If $x_{n} \stackrel{d, E}{\longrightarrow} x$ and $y_{n} \stackrel{d, E}{\longrightarrow} y$, then $d\left(x_{n}, y_{n}\right) \stackrel{o}{\rightarrow} d(x, y)$; that is, the vector metric map $d$ from $X^{2}$ to $E$ is vectorially continuous. Here, $X^{2}$ is equipped with the $E$-valued vector metric $\widetilde{d}$ defined as $\tilde{d}(z, w)=d\left(x_{1}, x_{2}\right)+d\left(y_{1}, y_{2}\right)$ for all $z=\left(x_{1}, y_{1}\right), w=$ $\left(x_{2}, y_{2}\right) \in X^{2}$, and $E$ is equipped with the absolute valued vector metric $|\cdot|$.

We recall that a subset $U$ of a vector metric space $(X, d, E)$ is called $E$-closed (or vector closed) whenever $\left(x_{n}\right) \subseteq U$ and $x_{n} \stackrel{d, E}{\longrightarrow} x$ imply $x \in U$.

Theorem 7. Let $(X, d, E)$ and $(Y, \rho, F)$ be vector metric spaces. If a function $f: X \rightarrow Y$ is vectorially continuous, then for every F-closed subset $B$ of $Y$ the set $f^{-1}(B)$ is E-closed in $X$.

Proof. For any $x \in f^{-1}(B)$, there exists a sequence $\left(x_{n}\right)$ in $f^{-1}(B)$ such that $x_{n} \stackrel{d, E}{\longrightarrow} x$. Since the function $f$ is vectorially continuous, $f\left(x_{n}\right) \stackrel{\rho, F}{\longrightarrow} f(x)$. But the set $B$ is $F$-closed, so $x \in$ $f^{-1}(B)$. Then, the set $f^{-1}(B)$ is $E$-closed.

If $E$ and $F$ are two Riesz spaces, then $E \times F$ is also a Riesz space with coordinatewise ordering defined as

$$
\left(e_{1}, f_{1}\right) \leq\left(e_{2}, f_{2}\right) \Longleftrightarrow e_{1} \leq e_{2}, f_{1} \leq f_{2}
$$

for all $\left(e_{1}, f_{1}\right),\left(e_{2}, f_{2}\right) \in E \times F$. The Riesz space $E \times F$ is a vector metric space, equipped with the biabsolute valued vector metric $|\cdot|$ defined as

$$
|a-b|=\left(\left|e_{1}-e_{2}\right|,\left|f_{1}-f_{2}\right|\right)
$$

for all $a=\left(e_{1}, f_{1}\right), b=\left(e_{2}, f_{2}\right) \in E \times F$. 
Let $d$ and $\rho$ be two vector metrics on $X$ which are $E$ valued and $F$-valued, respectively. Then the map $\delta$ defined as

$$
\delta(x, y)=(d(x, y), \rho(x, y))
$$

for all $x, y \in X$ is an $E \times F$-valued vector metric on $X$. We will call $\delta$ double vector metric.

Example 8. Let $(X, d, E)$ and $(X, \rho, F)$ be vector metric spaces.

(i) Suppose that $f: X \rightarrow E$ and $g: X \rightarrow F$ are vectorially continuous functions. Then the function $h$ from $X$ to $E \times F$ defined by $h(x)=(f(x), g(x))$ for all $x \in X$ is vectorially continuous with the double vector metric $\delta$ and the biabsolute valued vector metric $|\cdot|$.

(ii) Let $p_{E}: E \times F \rightarrow E$ and $p_{F}: E \times F \rightarrow F$ be the projection maps. Any function $h: X \rightarrow E \times F$ can be written as $h(x)=(f(x), g(x))$ for all $x \in X$ where $f=$ $p_{E} h$ and $g=p_{F} h$. If $h$ is vectorially continuous, so are $f$ and $g$ since $p_{E}$ and $p_{F}$ are vectorially continuous.

Let $(X, d, E)$ and $(Y, \rho, F)$ be vector metric spaces. Then $X \times Y$ is a vector metric space, equipped with the $E \times F$-valued product vector metric $\pi$ defined as

$$
\pi(z, w)=\left(d\left(x_{1}, x_{2}\right), \rho\left(y_{1}, y_{2}\right)\right)
$$

for all $z=\left(x_{1}, y_{1}\right), w=\left(x_{2}, y_{2}\right) \in X \times Y$.

Consider the vector metric space $(X \times Y, \pi, E \times F)$. The projection maps $p_{X}$ and $p_{Y}$ defined on $X \times Y$ which are $X$ valued and $Y$-valued, respectively, are vectorially continuous. For any function $f$ from a vector metric space $(Z, \delta, G)$ to $(X \times Y, \pi, E \times F)$, the function $f$ is vectorially continuous if and only if both $p_{X} f$ and $p_{Y} f$ are vectorially continuous.

Example 9. Let $(X, d, E)$ and $(Y, \rho, E)$ be vector metric spaces. Suppose that $f: X \rightarrow E$ and $g: Y \rightarrow E$ are vectorially continuous functions. Then the function $h$ from $X \times Y$ to $E$ defined by $h(x, y)=|f(x)-g(y)|$ for all $x \in X, y \in Y$ is vectorially continuous with the product vector metric $\pi$ and the absolute valued vector metric $|\cdot|$.

Example 10. Let $(X, d, E)$ and $(Y, \rho, F)$ be vector metric spaces. Suppose that $f: X \rightarrow E$ and $g: Y \rightarrow F$ are vectorially continuous functions. Then the function $h$ from $X \times Y$ to $E \times F$ defined by $h(x, y)=(f(x), g(y))$ for all $x \in X, y \in Y$ is vectorially continuous with the product vector metric $\pi$ and the biabsolute valued vector metric $|\cdot|$.

The last three examples inspire the following results.

Corollary 11. (a) If $f:(X, d, E) \rightarrow(Y, \eta, G)$ and $g:$ $(X, \rho, F) \rightarrow(Z, \xi, H)$ are vectorially continuous functions, then the function $h$ from $X$ to $Y \times Z$ defined by $h(x)=$ $(f(x), g(x))$ for all $x \in X$ is vectorially continuous with the $E \times F$-valued double vector metric $\delta$ and the $G \times H$-valued product vector metric $\pi$.

(b) Let $G$ be a Riesz space. If $f:(X, d, E) \rightarrow G$ and $g$ : $(Y, \rho, F) \rightarrow G$ are vectorially continuous functions, then the function $h$ from $X \times Y$ to $G$ defined by $h(x, y)=|f(x)-g(y)|$ for all $x \in X, y \in Y$ is vectorially continuous with the $E \times F$ valued product vector metric $\pi$ and the absolute valued vector metric $|\cdot|$.

(c) If $f:(X, d, E) \rightarrow(Z, \eta, G)$ and $g:(Y, \rho, F) \rightarrow$ $(W, \xi, H)$ are vectorially continuous functions, then the function $h$ from $X \times Y$ to $Z \times W$ defined by $h(x, y)=(f(x), g(y))$ for all $x \in X, y \in Y$ is vectorially continuous with the $E \times F$ valued and $G \times H$-valued product vector metrics.

We have the next proposition for any product vector metric.

Proposition 12. Let $\left(z_{n}\right)=\left(x_{n}, y_{n}\right)$ be a sequence in $(X \times$ $Y, \pi, E \times F)$ and let $z=(x, y) \in X \times Y$. Then, $z_{n} \stackrel{\pi, E \times F}{\longrightarrow} z$ if and only if $x_{n} \stackrel{d, E}{\longrightarrow} x$ and $y_{n} \stackrel{\rho, F}{\longrightarrow} y$.

Now let us give relevance between vectorial continuity of a function and closeness of its graph.

Corollary 13. Let $(X, d, E)$ and $(Y, \rho, F)$ be vector metric spaces and let $f$ be a function from $X$ to $Y$. For the graph $G_{f}$ of $f$, the following statements hold.

(a) The graph $G_{f}$ is $E \times F$-closed in $(X \times Y, \pi, E \times F)$ if and only if for every sequence $\left(x_{n}\right)$ with $x_{n} \stackrel{d, E}{\longrightarrow} x$ and $f\left(x_{n}\right) \stackrel{\rho, F}{\longrightarrow} y$ we have $y=f(x)$.

(b) If the function $f$ is vectorially continuous, then the graph $G_{f}$ is $E \times F$-closed.

(c) If the function $f$ is vectorially continuous at $x_{0} \in X$, then the induced function $h: X \rightarrow G_{f}$ defined by $h(x)=(x, f(x))$ is vectorially continuous at $x_{0} \in X$.

Proof. For the proof of (a), suppose that the graph $G_{f}$ is $E \times F$-closed. If $x_{n} \stackrel{d, E}{\longrightarrow} x$ and $f\left(x_{n}\right) \stackrel{\rho, F}{\longrightarrow} y$, then we have $\left(x_{n}, f\left(x_{n}\right)\right) \stackrel{\pi, E \times F}{\longrightarrow}(x, y)$ by Proposition 12 . Hence $(x, y) \in$ $G_{f}$, and so $y=f(x)$. Conversely, suppose that $\left(z_{n}\right)=$ $\left(x_{n}, f\left(x_{n}\right)\right)$ is a sequence in $G_{f}$ such that $z_{n} \stackrel{\pi, E \times F}{\longrightarrow} z=$ $(x, y) \in X \times Y$. By Proposition 12, $x_{n} \stackrel{d, E}{\longrightarrow} x$ and $f\left(x_{n}\right) \stackrel{\rho, F}{\longrightarrow} y$. Then $y=f(x)$, and so $z \in G_{f}$.

Proofs of (b) and (c) are similar to the proof of (a).

\section{Fundamental Vector Valued Function Classes}

Definition 14. The $E$-valued vector metric $d$ and $F$-valued vector metric $\rho$ on $X$ are said to be $(E, F)$-equivalent if for any $x \in X$ and any sequence $\left(x_{n}\right)$ in $X$

$$
x_{n} \stackrel{d, E}{\longrightarrow} x \text { iff } x_{n} \stackrel{\rho, F}{\longrightarrow} x .
$$

Lemma 15. For any two E-valued vector metrics $d$ and $\rho$ on $X$, the following statements are equivalent.

(a) There exist some $\alpha, \beta>0$ in $\mathbb{R}$ such that $\alpha d(x, y) \leq$ $\rho(x, y) \leq \beta d(x, y)$ for all $x, y \in X$. 
(b) There exist two positive and $\sigma$-order continuous operators $T$ and $S$ from $E$ to itself such that $\rho(x, y) \leq$ $T(d(x, y))$ and $d(x, y) \leq S(\rho(x, y))$ for all $x, y \in X$.

Proof. Let $T$ and $S$ be two operators defined as $T(a)=\beta a$ and $S(a)=\alpha^{-1} a$ for all $a \in E$. If (a) holds, then $T$ and $S$ are positive and $\sigma$-order continuous operators and satisfy $\rho(x, y) \leq T(d(x, y))$ and $d(x, y) \leq S(\rho(x, y))$ for all $x, y \in$ $X$. Conversely, (a) holds since every $(\sigma$-)order continuous operator is order bounded $[1,1.54]$.

Now, we give the following result for the equivalence of vector metrics.

Theorem 16. An E-valued vector metric $d$ and an F-valued vector metric $\rho$ on $X$ are $(E, F)$-equivalent if there exist positive and $\sigma$-order continuous two operators $T: E \rightarrow F, S: F \rightarrow E$ such that

$$
\rho(x, y) \leq T(d(x, y)), \quad d(x, y) \leq S(\rho(x, y))
$$

for all $x, y \in X$.

Example 17. Suppose that the ordering of $\mathbb{R}^{2}$ is coordinatewise.

(a) Let $d$ and $\rho$ be $\mathbb{R}$-valued and $\mathbb{R}^{2}$-valued vector metrics on $\mathbb{R}$, respectively, defined as

$d(x, y)=a|x-y|, \quad \rho(x, y)=(b|x-y|, c|x-y|)$,

where $b, c \geq 0$ and $a, b+c>0$. Consider the two operators $T: \mathbb{R} \rightarrow \mathbb{R}^{2} ; T(x)=a^{-1}(b x, c x)$ and $S: \mathbb{R}^{2} \rightarrow \mathbb{R} ; S(x, y)=a b^{-1} x$ for all $x, y \in \mathbb{R}$. Then, the operators $T$ and $S$ are positive and $\sigma$-order continuous, and (6) is satisfied. Hence, the metrics $d$ and $\rho$ are $\left(\mathbb{R}, \mathbb{R}^{2}\right)$-equivalent on $\mathbb{R}$.

(b) Let $d$ and $\rho$ be $\mathbb{R}$-valued and $\mathbb{R}^{2}$-valued vector metrics on $\mathbb{R}^{2}$, respectively, defined as

$$
\begin{aligned}
& d(x, y)=a\left|x_{1}-y_{1}\right|+b\left|x_{2}-y_{2}\right|, \\
& \rho(x, y)=\left(c\left|x_{1}-y_{1}\right|, e\left|x_{2}-y_{2}\right|\right),
\end{aligned}
$$

where $x=\left(x_{1}, x_{2}\right), y=\left(y_{1}, y_{2}\right)$, and $a, b, c, e>0$. Let $T: \mathbb{R} \rightarrow \mathbb{R}^{2}$ and $S: \mathbb{R}^{2} \rightarrow \mathbb{R}$ be two operators defined as $T(x)=\left(c a^{-1} x, e b^{-1} x\right)$ and $S(x, y)=$ $a c^{-1} x+b e^{-1} y$. Then, the operators $T$ and $S$ are positive and $\sigma$-order continuous. The condition (6) is satisfied. So, the vector metrics $d$ and $\rho$ are $\left(\mathbb{R}, \mathbb{R}^{2}\right)$-equivalent on $\mathbb{R}^{2}$. On the other hand, if $\eta$ is another $\mathbb{R}$-valued vector metric on $\mathbb{R}^{2}$ defined as

$$
\eta(x, y)=\max \left\{a\left|x_{1}-y_{1}\right|, b\left|x_{2}-y_{2}\right|\right\},
$$

where $x=\left(x_{1}, x_{2}\right), y=\left(y_{1}, y_{2}\right)$, and $a, b>0$, and the operator $S$ is defined as $S(x, y)=\max \left\{a c^{-1} x, b e^{-1} y\right\}$, then the vector metrics $\eta$ and $\rho$ are $\left(\mathbb{R}, \mathbb{R}^{2}\right)$-equivalent on $\mathbb{R}^{2}$.
Remark 18. Vectorial continuity is invariant under equivalent vector metrics.

Let us show how an isometry is defined between two vector metric spaces.

Definition 19. Let $(X, d, E)$ and $(Y, \rho, F)$ be vector metric spaces. A function $f: X \rightarrow Y$ is said to be a vector isometry if there exists a linear operator $T_{f}: E \rightarrow F$ satisfying the following two conditions:

(i) $T_{f}(d(x, y))=\rho(f(x), f(y))$ for all $x, y \in X$,

(ii) $T_{f}(a)=0$ implies $a=0$ for all $a \in E$.

If the function $f$ is onto, and the operator $T_{f}$ is a lattice homomorphism, then the vector metric spaces $(X, d, E)$ and $\left(Y, \rho, T_{f}(E)\right)$ are called vector isometric.

Remark 20. A vector isometry is a vectorial distance preserving one-to-one function.

Example 21. Let $d$ be $\mathbb{R}$-valued vector metric and let $\rho$ be $\mathbb{R}^{2}$ valued vector metric on $\mathbb{R}$ defined as

$$
d(x, y)=a|x-y|, \quad \rho(x, y)=(b|x-y|, c|x-y|),
$$

where $b, c \geq 0$ and $a, b+c>0$. Consider the identity mapping $I$ on $\mathbb{R}$ and the operator $T_{I}: \mathbb{R} \rightarrow \mathbb{R}^{2}$ defined as $T_{I}(x)=a^{-1}(b x, c x)$ for all $x \in \mathbb{R}$. Then, the identity mapping $I$ is a vector isometry. So, the vector metric spaces $(\mathbb{R}, d, \mathbb{R})$ and $(\mathbb{R}, \rho,\{(x, y): c x=b y ; x, y \in \mathbb{R}\})$ are vector isometric.

Definition 22. Let $(X, d, E)$ and $(Y, \rho, F)$ be vector metric spaces. A function $f: X \rightarrow Y$ is said to be a vector homeomorphism if $f$ is one-to-one and vectorially continuous and has a vectorially continuous inverse on $f(X)$. If the function $f$ is onto, then the vector metric spaces $X$ and $Y$ are called vector homeomorphic.

Remark 23. A vector homeomorphism is one-to-one function that preserves vectorial convergence of sequences.

By Theorem 7, we can develop another characterization result for vector homeomorphisms.

Theorem 24. An onto vector homeomorphism is a one-to-one function that preserves vector closed sets.

Proof. Let $f: X \rightarrow Y$ be an $(E, F)$-homeomorphism. Since $f$ is a one-to-one function and its inverse $f^{-1}$ is vectorially continuous, by Theorem 7 for every $E$-closed set $A$ in $X$, $f(A)=\left(f^{-1}\right)^{-1}(A)$ is $F$-closed in $Y$.

The following example illustrates a relationship between vectorial equivalence and vector homeomorphism.

Example 25. Let $d$ and $\rho$ be two $(E, F)$-equivalent vector metrics on $X$. Then the vector metric spaces $(X, d, E)$ and $(X, \rho, F)$ are vector homeomorphic under the identity mapping. On the other hand, if any two vector metric spaces 
$(X, d, E)$ and $(Y, \rho, F)$ are vector homeomorphic under a function $f$, then the vector metrics $d$ and $\delta$ defined as

$$
\delta(x, y)=\rho(f(x), f(y))
$$

for all $x, y \in X$ are $(E, F)$-equivalent vector metrics on $X$.

\section{Extension Theorems on Continuity}

If $X$ and $Y$ are vector metric spaces, $A \subseteq X$, and $f: A \rightarrow Y$ is vectorially continuous, then we might ask whether there exists a vectorial continuous extension $g$ of $f$. Below, we deal with some simple extension techniques.

Theorem 26. Let $(X, d, E)$ and $(Y, \rho, F)$ be vector metric spaces, and let $f$ and $g$ be vectorially continuous functions from $X$ to $Y$. Then the set $\{x \in X: f(x)=g(x)\}$ is an E-closed subset of $X$.

Proof. Let $B=\{x \in X: f(x)=g(x)\}$. Suppose $\left(x_{n}\right) \subseteq B$ and $x_{n} \stackrel{d, E}{\longrightarrow} x$. Since $f$ and $g$ are vectorially continuous, there exist sequences $\left(a_{n}\right)$ and $\left(b_{n}\right)$ such that $a_{n} \downarrow 0, b_{n} \downarrow 0$ and $\rho\left(f\left(x_{n}\right), f(x)\right) \leq a_{n}, \rho\left(g\left(x_{n}\right), g(x)\right) \leq b_{n}$ for all $n$. Then

$$
\begin{gathered}
\rho(f(x), g(x)) \leq \rho\left(f\left(x_{n}\right), f(x)\right)+\rho\left(f\left(x_{n}\right), g\left(x_{n}\right)\right) \\
+\rho\left(g\left(x_{n}\right), g(x)\right) \leq a_{n}+b_{n}
\end{gathered}
$$

for all $n$. So $f(x)=g(x)$; that is, $x \in B$. Hence, the set $B$ is an $E$-closed subset of $X$.

The following corollary is a consequence of Theorem 26.

Corollary 27. Let $(X, d, E)$ and $(Y, \rho, F)$ be vector metric spaces and let $f$ and $g$ be vectorially continuous functions from $X$ to $Y$. If the set $\{x \in X: f(x)=g(x)\}$ is E-dense in $X$, then $f=g$.

Definition 28. Let $(X, d, E)$ and $(Y, \rho, F)$ be vector metric spaces.

(a) A function $f: X \rightarrow Y$ is said to be topological uniformly continuous on $X$ if for every $b>0$ in $F$ there exist some $a$ in $E$ such that for all $x, y \in X$, $\rho(f(x), f(y))<b$ whenever $d(x, y)<a$.

(b) A function $f: X \rightarrow Y$ is said to be vectorial uniformly continuous on $X$ if for every $E$-Cauchy sequence $\left(x_{n}\right)$ the sequence $f\left(x_{n}\right)$ is F-Cauchy.

Theorem 29. Let $(X, d, E)$ and $(Y, \rho, F)$ be vector metric spaces where $F$ is Archimedean. If a function $f: X \rightarrow Y$ is topological uniformly continuous, then $f$ is vectorial uniformly continuous.

Proof. Suppose that $\left(x_{n}\right)$ is an $E$-Cauchy sequence. Then there exists a sequence $\left(a_{n}\right)$ in $E$ such that $a_{n} \downarrow 0$ and $d\left(x_{n}, x_{n+p}\right) \leq$ $a_{n}$ for all $n$ and $p$. Let $b$ be any nonzero positive element in $F$. By topological uniform continuity of $f$, there exist some $b_{n}=b_{n}((1 / n) b)$ in $E$ such that the inequality $d\left(x_{n}, x_{n+p}\right)<b_{n}$ implies $\rho\left(f\left(x_{n}\right), f\left(x_{n+p}\right)\right)<(1 / n) b$ for all $n$ and $p$. Then there exist elements $c_{n}=a_{n} \vee b_{n}$ in $E$ such that $d\left(x_{n}, x_{n+p}\right) \leq a_{n}<c_{n}$ implies $\rho\left(f\left(x_{n}\right), f\left(x_{n+p}\right)\right)<(1 / n) b$ for all $n$ and $p$. Since $F$ is Archimedean, $(1 / n) b \downarrow 0$. Hence, the sequence $f\left(x_{n}\right)$ is $F$ Cauchy.

Example 30. (a) For a vector isometry $f$ between two vector metric spaces $(X, d, E)$ and $(Y, \rho, F)$, the function $f$ is vectorial uniformly continuous if $T_{f}$ is positive and $\sigma$-order continuous.

(b) For an element $y$ in a vector metric space $(X, d, E)$, the function $f_{y}: X \rightarrow E$ defined by $f_{y}(x)=d(x, y)$ for all $x \in X$ is vectorial uniformly continuous.

(c) For a subset $A$ of a vector metric space $(X, d, E)$ where $E$ is Dedekind complete, the function $f_{A}: X \rightarrow E$ defined by $f_{A}(x)=d(x, A)=\inf \{d(x, y): y \in A\}$ for all $x \in X$ is vectorial uniformly continuous.

The following theorem enables us to establish an extension property for the functions between vector metric spaces.

Theorem 31. Let $A$ be E-dense subset of a vector metric space $(X, d, E)$ and let $(Y, \rho, F)$ be an $F$-complete vector metric space where $F$ is Archimedean. If $f: A \rightarrow Y$ is a topological uniformly continuous function, then $f$ has a unique vectorially continuous extension to $X$ which is also topological uniformly continuous.

Proof. Let $x \in X$. Then there exists a sequence $\left(x_{n}\right)$ in $A$ such that $x_{n} \stackrel{d, E}{\longrightarrow} x$. Since the function $f$ is vectorial uniformly continuous on $A$ by Theorem 29, the sequence $\left(f\left(x_{n}\right)\right)$ is $F$ Cauchy in $F$-complete vector metric space $Y$. Hence, there exists an element $y \in Y$ such that $f\left(x_{n}\right) \stackrel{\rho, F}{\longrightarrow} y$. Define an extension $g$ of $f$ on $X$ by $g(x)=y$. This extension is well-defined; that is, the value of $g$ at $x$ is independent of the particular sequence $\left(x_{n}\right)$ chosen $E$-convergent to $x$. We need to show that $g$ is topological uniformly continuous on $X$.

Let $a>0$ in $F$. Choose $b>0$ in $E$ such that for $x, y \in A d(x, y)<b$ implies $\rho(f(x), f(y))<a$. Let $x, y \in X$ satisfy $d(x, y)<b$. Choose two sequences $\left(x_{n}\right)$ and $\left(y_{n}\right)$ in $A$ such that $x_{n} \stackrel{d, E}{\longrightarrow} x$ and $y_{n} \stackrel{d, E}{\longrightarrow} y$. Then, $d\left(x_{n}, y_{n}\right) \stackrel{o}{\rightarrow} d(x, y)$ in $E$. Fix $n_{0}$ such that $d\left(x_{n}, y_{n}\right)<b$ for all $n>n_{0}$. Then $\rho\left(f\left(x_{n}\right), f\left(y_{n}\right)\right)<a$ for all $n>n_{0}$. By the vectorial uniform continuity of $f, f\left(x_{n}\right)$ and $f\left(y_{n}\right)$ are $F$ Cauchy sequences in $Y$. Since $Y$ is $F$-complete, there exist two points $u$ and $v$ in $Y$ such that $f\left(x_{n}\right) \stackrel{\rho, F}{\longrightarrow} u$ and $f\left(y_{n}\right) \stackrel{\rho, F}{\longrightarrow} v$. By the definition of the function $g$, we have $g(x)=u$ and $g(y)=v$. Then $\rho\left(g\left(x_{n}\right), g\left(y_{n}\right)\right) \stackrel{o}{\rightarrow} \rho(g(x), g(y))$ in $F$, and therefore, $\rho(g(x), g(y)) \leq b$. This shows that $g$ is a topological uniformly continuous function on $X$.

\section{Vectorially Continuous Function Spaces}

Definition 32. Let $X$ be any nonempty set and let $(Y, \rho, F)$ be a vector metric space. Then a sequence $\left(f_{n}\right)$ of functions from $X$ to $Y$ is said to be uniformly $F$-convergent to a function $f$ : $X \rightarrow Y$, if there exists a sequence $\left(a_{n}\right)$ in $F$ such that $a_{n} \downarrow 0$ and $\rho\left(f_{n}(x), f(x)\right) \leq a_{n}$ holds for all $x \in X$ and all $n \in \mathbb{N}$. 
Now we give the uniform limit theorem in vector metric spaces.

Theorem 33. Let $\left(f_{n}\right)$ be a sequence of vectorially continuous functions between two vector metric spaces $(X, d, E)$ and $(Y, \rho, F)$. If $\left(f_{n}\right)$ is uniformly $F$-convergent to $f$, then the function $f$ is vectorially continuous.

Proof. Let $\left(x_{n}\right) \subseteq X$ such that $x_{n} \stackrel{d, E}{\longrightarrow} x$ in $X$. Since $\left(f_{n}\right)$ is uniformly $F$-convergent to $f$, there is a sequence $\left(a_{n}\right)$ in $F$ such that $a_{n} \downarrow 0$ and $\rho\left(f_{n}(x), f(x)\right) \leq a_{n}$ for all $n \in \mathbb{N}$. For each $k \in \mathbb{N}$, there is a sequence $\left(b_{n}\right)$ in $F$ such that $b_{n} \downarrow 0$ and $\rho\left(f_{k}\left(x_{n}\right), f_{k}(x)\right) \leq b_{n}$ for all $n \in \mathbb{N}$ by the vectorial continuity of $f_{k}$. Note that for $k=n$

$$
\begin{aligned}
\rho\left(f\left(x_{n}\right), f(x)\right) \leq & \rho\left(f\left(x_{n}\right), f_{n}\left(x_{n}\right)\right)+\rho\left(f(x), f_{n}(x)\right) \\
& +\rho\left(f_{n}\left(x_{n}\right), f_{n}(x)\right) \leq 2 a_{n}+b_{n} .
\end{aligned}
$$

This implies $f\left(x_{n}\right) \stackrel{\rho, F}{\longrightarrow} f(x)$.

Let $A$ be a nonempty subset of a vector metric space $(X, d, E)$. E-diameter of $A, d(A)$, is defined as $\sup \{d(x, y)$ : $x, y \in A\}$. The set $A$ is called E-bounded if there exists an element $a>0$ in $E$ such that $d(x, y) \leq a$ for all $x, y \in A$. Every $E$-bounded subset of $X$ has an $E$-diameter whenever $E$ is Dedekind complete.

Definition 34. A function $f: X \rightarrow Y$ between two vector metric spaces $(X, d, E)$ and $(Y, \rho, F)$ is called vectorial bounded if $f$ maps $E$-bounded subsets of $X$ to $F$-bounded subsets of $Y$.

Theorem 35. A function $f: X \rightarrow Y$ between two vector metric spaces $(X, d, E)$ and $(Y, \rho, F)$ is vectorial bounded if there exists a positive operator $T: E \rightarrow F$ such that $\rho(f(x), f(y)) \leq T(d(x, y))$ for all $x, y \in X$.

Let $C_{v}(X, F)$ and $C_{t}(X, F)$ be the collections of all vectorially continuous and topologically continuous functions between a vector metric space $(X, d, E)$ and a Riesz space $F$, respectively. By Theorem $4, C_{t}(X, F) \subseteq C_{v}(X, F)$ whenever $F$ is Archimedean.

Theorem 36. The spaces $C_{v}(X, F)$ and $C_{t}(X, F)$ are Riesz spaces with the natural partial ordering defined as $f \leq g$ whenever $f(x) \leq g(x)$ for all $x \in X$.

Consider an $E$-bounded vector metric space $X$ and a Dedekind complete Riesz space $F$. Let $C_{v}^{o}(X, F)$ be a subset of $C_{v}(X, F)$ such that, for any $f$ in $C_{v}^{o}(X, F)$, there is a positive operator $T: E \rightarrow F$ satisfying $|f(x)-f(y)| \leq T(d(x, y))$ for all $x, y \in X$. Since the Birkhoff inequality $([1,1.9(2)] ;[2$, 12.4(ii)])

$$
|f \vee g(x)-f \vee g(y)| \leq|f(x)-f(y)|+|g(x)-g(y)|
$$

holds for all $x, y \in X$, then the subset $C_{v}^{o}(X, F)$ is a Riesz subspace of $C_{v}(X, F)$. By Theorem 35, every $f \in C_{v}^{o}(X, F)$ is vectorial bounded function. This argument gives us the following result.

Corollary 37. The subset $C_{v}^{o}(X, F)$ described above is a vector metric space equipped with the $F$-valued uniform vector metric defined as $d_{\infty}(f, g)=\sup _{x \in X}|f(x)-g(x)|$.

\section{Conflict of Interests}

The author declares that there is no conflict of interests regarding the publication of this paper.

\section{References}

[1] C. D. Aliprantis and O. Burkinshaw, Positive Operators, Springer, Dordrecht, The Netherlands, 2006.

[2] W. A. J. Luxemburg and A. C. Zaanen, Riesz Space I, NorthHolland, Amsterdam, The Netherland, 1971.

[3] C. Çevik and I. Altun, "Vector metric spaces and some properties," Topological Methods in Nonlinear Analysis, vol. 34, no. 2, pp. 375-382, 2009.

[4] I. Altun and C. Çevik, "Some common fixed point theorems in vector metric spaces," Filomat, vol. 25, no. 1, pp. 105-113, 2011.

[5] Z. Páles and I.-R. Petre, "Iterative fixed point theorems in Emetric spaces," Acta Mathematica Hungarica, vol. 140, no. 1-2, pp. 134-144, 2013.

[6] I.-R. Petre, "Fixed point theorems in vector metric spaces for single-valued operators," in Annals of the Tiberiu Popoviciu Seminar of Functional Equations, Approximation and Convexity, vol. 9, pp. 59-80, 2011.

[7] I.-R. Petre, "Fixed points for $\varphi$-contractions in $E$-Banach spaces," Fixed Point Theory, vol. 13, no. 2, pp. 623-640, 2012.

[8] P. P. Zabrejko, " $K$-metric and $K$-normed linear spaces: survey," Universitat de Barcelona: Collectanea Mathematica, vol. 48, no. 4-6, pp. 825-859, 1997. 


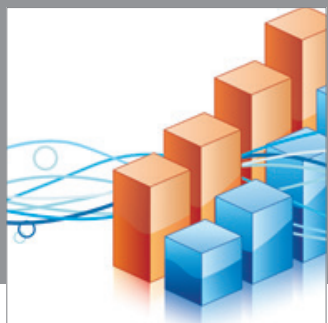

Advances in

Operations Research

mansans

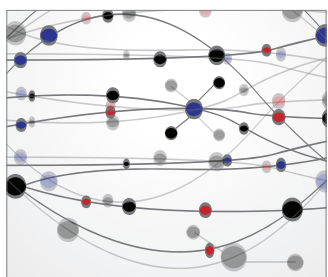

The Scientific World Journal
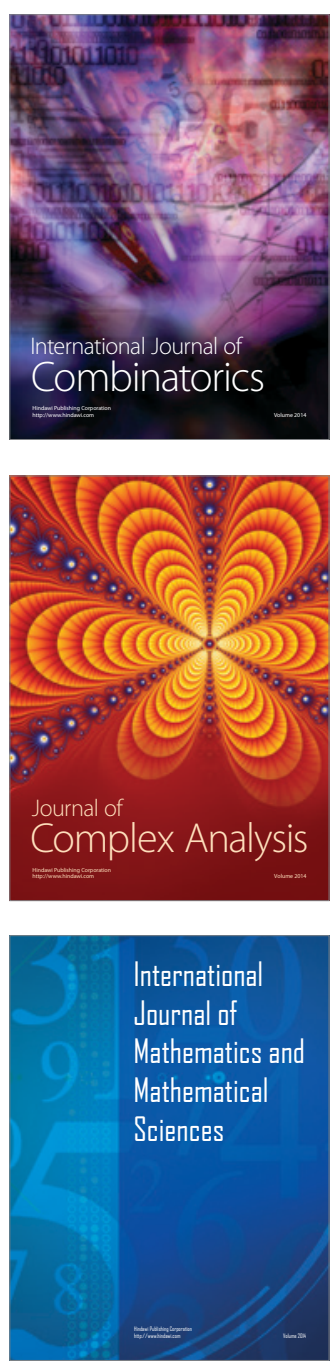
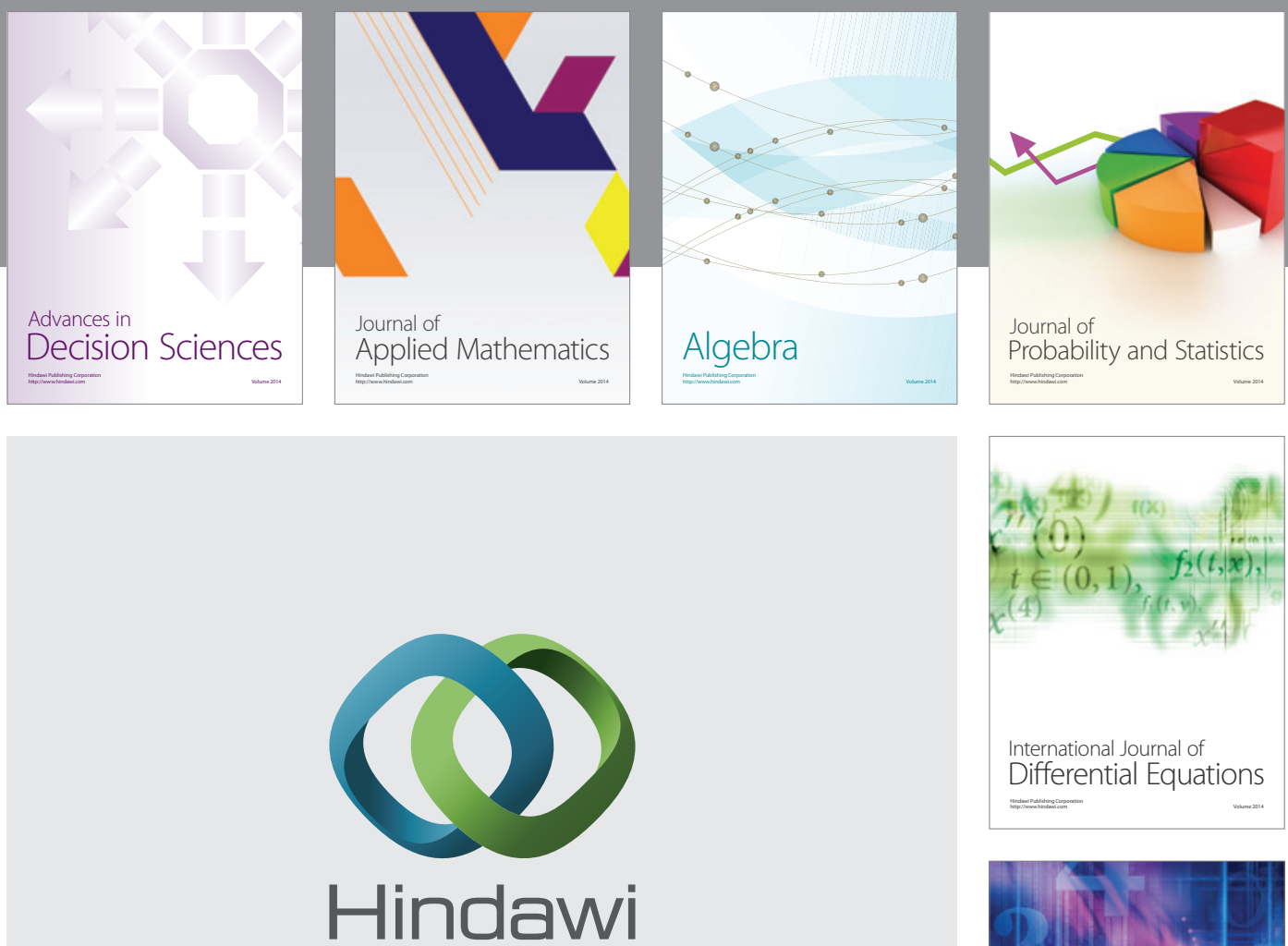

Submit your manuscripts at http://www.hindawi.com
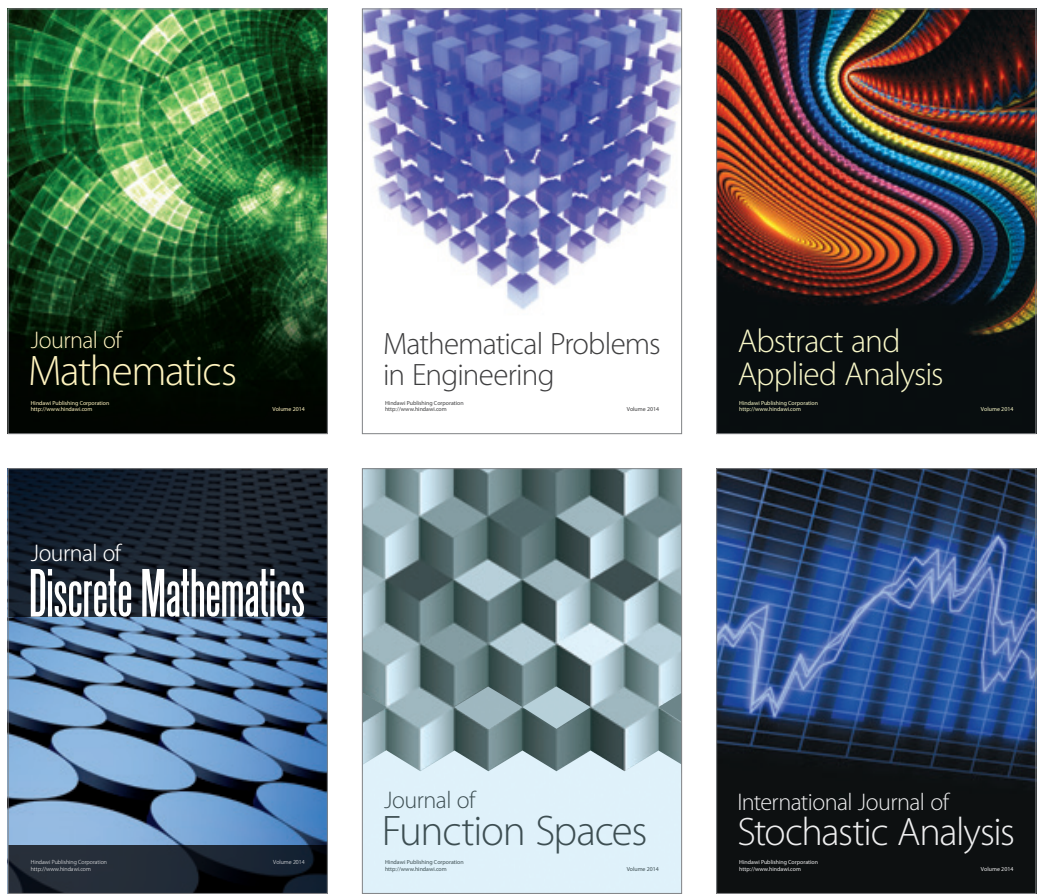

Journal of

Function Spaces

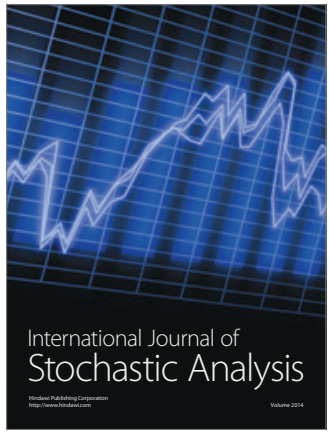

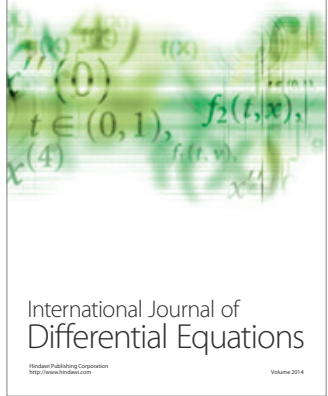
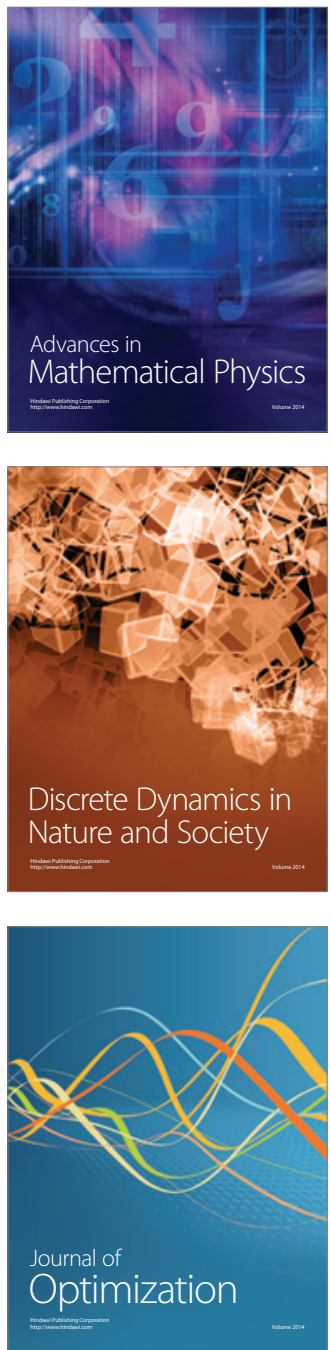